\title{
Focal Fatty Areas in the Myocardium of Patients With Tuberous Sclerosis Complex
}

\section{A Unique Finding}

\author{
Miraude E.A.P.M. Adriaensen, MD, MSc, ${ }^{*} \dagger$ Harm H.H. Feringa, MD, PhD, $\neq$ \\ Cornelia M. Schaefer-Prokop, MD, PhD, $\S$ Sandra A.P. Cornelissen, MD, MSc, $\dagger$ \\ Bernard A. Zonnenberg, MD, PhD, /l and Mathias Prokop, MD, PhD $\uparrow$
}

\begin{abstract}
With this collection of computed tomography and magnetic resonance images, we illustrate a recently described novel finding in the myocardium of patients with tuberous sclerosis complex.
\end{abstract}

Key Words: tuberous sclerosis complex, heart, fat, myocardium, computed tomography, magnetic resonance imaging

( Thorac Imaging 2010;00:000-000)

W ith this collection of computed tomography (CT) and magnetic resonance images (MRIs), we illustrate a recently described finding in the myocardium of patients with tuberous sclerosis complex (TSC). TSC is an autosomaldominant neurocutaneous disorder characterized by tumor-like malformations involving many organ systems, including the brain (cortical tubers, subependymal nodules, and subependymal giant cell astrocytomas), the lungs (lymphangioleiomyomatosis), the kidneys (angiomyolipomas), and the skin (facial angiofibromas or forehead plaques, shagreen patches, and hypomelanotic macules). ${ }^{1}$

\section{CASE REPORT}

Within the field of cardiology, patients with TSC can present at a fetal or pediatric age due to the development of cardiac rhabdomyomas, which tend to regress over time. After childhood, patients with TSC who develop cardiac dysfunction suffer mostly from cardiac arrhythmias. ${ }^{2}$ In Figure 1, we show 2 different patients with TSC with focal circumscribed hypodense areas within the myocardium depicted on abdominal CT scans performed to monitor their renal angiomyolipomas. On MRI, these fatty foci show the signal intensity of fat (Fig. 2).

From the *Department of Radiology, Atrium Medical Center, Heerlen; Departments of $\dagger$ Radiology; ॥Internal Medicine, University Medical Center Utrecht, Utrecht; §Department of Radiology, Meander Medical Center, Amersfoort; $\uparrow^{-D e p a r t m e n t ~ o f ~ R a d i o l o g y, ~ R a d b o u d ~}$ University Nijmegen Medical Center, Nijmegen, The Netherlands; and \$Department of Epidemiology and Public Health, Yale University School of Medicine, New Haven, CT.

Disclosures: No conflicts to disclose.

Reprints: Miraude E.A.P.M. Adriaensen, MD, MSc, Department of Radiology, Atrium Medical Center, Henri Dunantstraat 5, 6419 PC Heerlen, The Netherlands (e-mail: miraude@gmail.com).

Copyright (C) 2010 by Lippincott Williams \& Wilkins
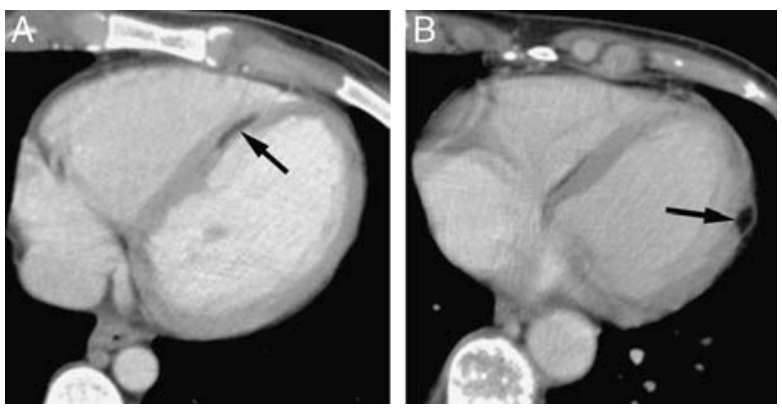

FIGURE 1. Fatty foci in the myocardium demonstrated on nongated contrast-enhanced abdominal CT scans in patients with TSC. A, Fatty focus in the interventricular septum (arrow) in a 25-year-old male patient. B, Fatty focus in the left ventricular wall (arrow) in a 59-year-old female patient.

\section{DISCUSSION}

In comparison with the known causes of fat in the myocardium (ie, epicardial fat deposits, old myocardial infarctions, arrhythmogenic right ventricular dysplasia, normal right ventricular fat, hemangiomas, and liposarcomas), the fatty foci in patients with TSC seem to have unique characteristics consisting of a combination of focality, well-circumscribed form, location into the midmyocardium, pure fat density, absence of enhancement,
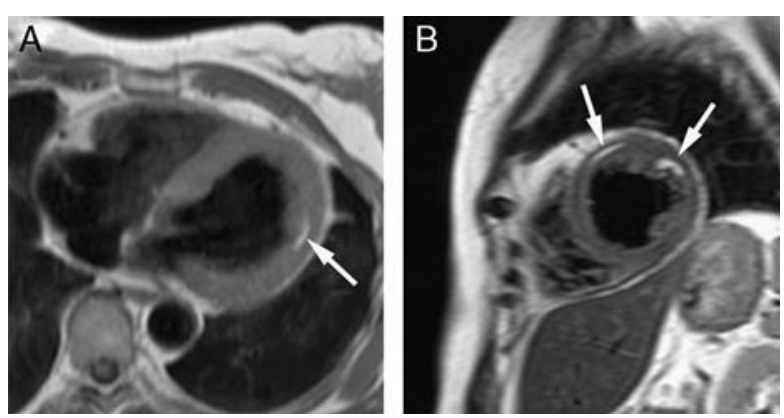

FIGURE 2. Fatty foci in the myocardium demonstrated on MRI in a 49-year-old female patient with TSC. A, Transverse dark-blood T1-weighted TSE image. Arrow points at fatty focus in the left ventricular wall. B, Short-axis dark-blood T2-weighted TSE image. Arrows point at fatty foci in the left ventricular wall. TSE indicates turbo spin echo. 
and absence of invasive behavior. ${ }^{3}$ In a recently published case-control study, the majority of patients with TSC showed these well-circumscribed foci of fat density in the myocardium on abdominal CT scans, which were not found in an age-matched and sex-matched control group without TSC. ${ }^{3}$ To our knowledge, no MRIs of these characteristic fatty foci in TSC have been published yet. On echocardiography, these fatty foci can be seen as areas of increased echogenicity. ${ }^{4}$ Therefore, if in daily practice one encounters these types of fatty foci in the myocardium on cardiac CT, cardiac MRI, or echocardiography, one should remember to look for other features of TSC.

\section{REFERENCES}

1. Crino PB, Nathanson KL, Henske EP. The tuberous sclerosis complex. $N$ Engl J Med. 2006;355:1345-1356.

2. Roach ES, DiMario FJ, Kandt RS, et al. Tuberous sclerosis consensus conference: recommendations for diagnostic evaluation. National tuberous sclerosis association. J Child Neurol. 1999;14:401-407.

3. Adriaensen MEAPM, Schaefer-Prokop CM, Duyndam DA, et al Fatty foci in the myocardium in patients with tuberous sclerosis complex: common finding at CT. Radiology. 2009;253:359-363.

4. Adriaensen MEAPM, Cramer MJM, Brouha MEE, et al Findings in screening echocardiography in patients with tuberous sclerosis complex. Tex Heart Inst J. 2010;37:280-283. 